\title{
Codimension Two Branes in Einstein-Gauss-Bonnet Gravity
}

\author{
Peng Wang $^{1 *}$ and Xin-He Meng ${ }^{1,2} \dagger$ \\ 1. Department of Physics, Nankai University, Tianjin, 300071, P.R.China \\ 2. CCAST (World Laboratory) P.O. Box 8730, Beijing 100080, China
}

\begin{abstract}
Codimension two branes play an interesting role in attacking the cosmological constant problem. Recently, in order to handle some problems in codimension two branes in Einstein gravity, Bostock et al. have proposed using six-dimensional Einstein-Gauss-Bonnet (EGB) gravity instead of sixdimensional Einstein gravity. In this paper, we present the solutions of codimension two branes in six-dimensional EGB gravity. We show that Einstein's equations take a "factorizable" form for a factorized metric tensor ansatz even in the presence of the higher-derivative Gauss-Bonnet term. Especially, a new feature of the solution is that the deficit angle depends on the brane geometry. We discuss the implication of the solution to the cosmological constant problem. We also comment on a possible problem of inflation model building on codimension two branes.
\end{abstract}

\footnotetext{
* E-mail: pewang@eyou.com

† E-mail: xhmeng@phys.nankai.edu.cn
} 


\section{INTRODUCTION}

The idea of braneworlds and large extra dimensions [1] implies that the cosmological constant problem (see Refs. 22, 3] for reviews) may be a clue of our unawareness of the true nature of spacetime: vacuum energy may be large, but it simply does not gravitate in the 4dimensional braneworld where we are living in. The key point is that, cosmological constant is a reflection of 4-dimensional spacetime geometry and thus is what is observed directly in cosmological observations. The puzzle arises only after we use General Relativity to find that cosmological constant describes vacuum energy of standard model particles. So if we modify gravity theory by introducing higher dimensional spacetime and objects like branes, it is possible that 4-dimensional cosmological constant is not linked to 4-dimensional vacuum energy, but something else such as higher dimensional vacuum energy. See, e.g., Refs. [4, 5] for some earlier endeavors in this direction.

Recently, Carroll and Guica presented an interesting exact solution of this type [6]. They considered a factorizable braneworld spacetime with two extra dimensions and explicit brane sources. The compactification manifold has the topology of a two-sphere, and is stabilized by both a bulk cosmological constant and a magnetic flux. From their solution, they found that the flat nature of the 4-dimensional geometry is independent of the brane tension. This feature moves the cosmological constant problem completely into the extra dimensions. Of course, this is not a complete solution to the cosmological constant problem, since it still needs fine-tuning in the bulk. But it transforms the nature of the problem in a suggestive way.

The interesting feature of Carroll and Guica's solution is not an accident (see Ref. 7] for some other models of codimension 2 branes that share the similar feature, see also Ref. [8] for earlier ideas along this line). It can be shown that the independence of 4-dimensional geometry on the brane tension is a general feature of codimension 2 branes in factorizable spacetime in Einstein gravity. The following discussions will also be helpful for us to understand the properties of codimension 2 branes in Einstein-Gauss-Bonnet gravity (see Sec (II).

Let's consider a factorizable metric ansatz,

$$
d s^{2}=G_{A B} d X^{A} d X^{B}=g_{\mu \nu}(x) d x^{\mu} d x^{\nu}+\gamma_{a b}(y) d y^{a} d y^{b}
$$

where $A, B=0, \ldots, 5, \mu, \nu=0, \ldots, 3$ and $a, b=4,5$. The $\gamma_{a b}$ is the metric of a Einstein 
manifold with curvature $k=-1,0,1$. Note that due to the presence of branes, there will be deficit angles in the extra dimensions at the positions of the branes (see Sec III), but this will not influence the local geometry of the extra dimensions at other points.

We will consider the simplest model of branes which is also the case considered in most of the literature on braneworld cosmology: the branes are described by Nambu-Goto action (see Ref.[9] for an elegant review),

$$
S_{N G}=\int d^{6} X \sqrt{|G|} \mathcal{L}_{\text {brane }}
$$

where

$$
\mathcal{L}_{\text {brane }}=-\sum_{i} \int d^{4} x \sqrt{\frac{|g|}{|G|}} \sigma_{i} \delta^{(6)}\left(X-X_{i}(x)\right),
$$

in which $i$ labels the branes, $\sigma_{i}$ and $X_{i}$ are the tension and position of the $i$ th brane, respectively. The energy-momentum tensor of branes follow by varying $G^{A B}$ in (2) [9]

$$
T_{A B}^{b}=-\sum_{i} \frac{\sigma_{i}}{\sqrt{\gamma}}\left(\begin{array}{cc}
g_{\mu \nu} & 0 \\
0 & 0
\end{array}\right) \delta^{(2)}\left(y-y_{i}\right)
$$

With the help of the fact that the Einstein tensor, $G_{a b}=R_{a b}-\frac{1}{2} h_{a b} R$, vanishes identically for any 2-dimensional metric $h_{a b}$ and denoting the bulk energy-momentum tensor by $T_{A B}^{B}$, contracting the transverse component of the Einstein equation gives

$$
R[g]=-\frac{2}{M_{6}^{4}} T_{2}^{B}
$$

where $T_{\gamma}^{B} \equiv T_{a b}^{B} \gamma^{a b}$; while contracting the longitudinal component gives

$$
R[\gamma]+\frac{1}{2} R[g]=-\frac{1}{2 M_{6}^{4}}\left[T_{g}^{B}+T^{b}\right] .
$$

where $T_{g}^{B} \equiv T_{\mu \nu}^{B} g^{\mu \nu}$ and $T^{b} \equiv T_{\mu \nu}^{b} g^{\mu \nu}$. Now, Eq. (15) tells us that the scalar curvature of the 4-dimensional spacetime is totally determined by the transverse component of the total bulk energy-momentum tensor. Thus if we assume the 4-dimensional geometry to be maximal symmetric, then it is determined totally by the transverse part of the bulk energy momentum tensor. Specifically, the 4-dimensional geometry does not depend on the brane tension. Then, after we find $R[g]$ from Eq.(15), substituting it into Eq.(6), we can find the bulk curvature $R[\gamma]$. In sum, for codimension 2 branes in factorizable spacetime, the brane geometry is determined by the transverse component of the Einstein equations and 
the bulk geometry is determined by the longitudinal component of the Einstein equations. Roughly speaking, we can say that Einstein equations in factorizable spacetime are also "factorizable". This is the secret of codimension 2 branes in Einstein gravity.

While the above discussion is exiting, unfortunately, when considering realistic cosmological evolution of this model, we will encounter some fundamental difficulties. One of them is that if we assume the brane energy-momentum tensor to be of the form as the perfect fluid, i.e. $T_{\nu}^{\mu}=\{\rho, p, p, p\} \delta_{\nu}^{\mu}$, then $\rho$ and $p$ must satisfy $\rho+p=0$, i.e. it behaves like the brane tension [10]. This forbids us adding dust and radiation on the brane, thus it is cosmologically unrealistic. To remedy this and other difficulties of codimension 2 branes in Einstein gravity, recently, Bostock et al. suggested that we may add the Gauss-Bonnet term to the 6-dimensional gravitational action [11] (however, see also Ref. [12] and reference therein for some other suggestions to handle this problem). It is also worth commenting that the idea that 6-dimensional Einstein-Gauss-Bonnet (EGB) gravity could be relevant in relation to the cosmological constant problem was originally presented in Ref.[13] (see also Ref. [14] for some subsequent related works). The Gauss-Bonnet term is quadratic in the curvature tensors and is a topological invariant in 4-dimensional manifold (see, e.g., Ref. 15]); but in higher dimensions, it has the well-know property that the equation of motion derived from it remains second order differential equations of the metric. Furthermore, considering higher derivative terms is also necessary to develop the braneworld scenario in a more string theoretic setting (see, e.g., Ref.[16]). Specifically, the Gauss-Bonnet combination arises as the leading order for quantum corrections in the heterotic string effective action and is the only quadratic combination of curvature tensors that is ghost-free [17].

Thus, the investigation of codimension 2 branes in EGB gravity is well motivated (see Ref.[18] for some other recent discussion of codimension 2 branes in EGB gravity). Of course, one of the best way to understand the property of a gravity theory is studying its exact solutions. Especially in the present case, the EGB gravity is intended to remedy the model in Einstein gravity. Thus one natural step is to derive and compare the corresponding solutions in EGB gravity under the same assumption of spacetime geometry and matter content with Einstein gravity case. In particular, it is important to check that the important property in Einstein gravity, i.e. the independence of the 4-dimensional geometry on the brane tension, is retained in EGB gravity. If this were not the case, considering EGB gravity would not be so well motivated. We will see in Sec II that the discussion above for Einstein 
gravity also applies to EGB gravity, thus EGB gravity retains the main features of Einstein gravity. In Sec III, we will also see that some new features will arise in EGB gravity. The last section, Sec IV is devoted to conclusions and we comment on inflation model building in the codimension 2 brane scenario.

\section{EINSTEIN-GAUSS-BONNET EQUATION IN FACTORIZABLE SPACETIME}

Let's consider adding the Gauss-Bonnet term to modify the 6-dimensional gravity [1]], which is described by the action

$$
S_{6}=\int d^{6} X \sqrt{|G|} \frac{M_{6}^{4}}{2}\left[R+\alpha R_{G B}^{2}\right]
$$

where $\alpha$ is the Gauss-Bonnet coupling constant with dimension $[\alpha]=(\text { mass })^{-2}$. Following the original derivation [17], one generally assumes $\alpha \geq 0$, but in the literature the $\alpha<0$ case is also often discussed. We will see in Sec III that, from the exact solution we found, the requirement of the geometry to be nonsingular will rule out a negative Gauss-Bonnet coupling constant. The Gauss-Bonnet term $R_{G B}$ is given by

$$
R_{\mathrm{GB}}^{2}=R^{2}-4 R^{A B} R_{A B}+R^{A B C D} R_{A B C D}
$$

Then the gravity field equation in 6-dimensional is described by the Einstein-GaussBonnet equation,

$$
G_{A B}+\alpha H_{A B}=\frac{1}{M_{6}^{4}} T_{A B}
$$

where

$$
H_{A B}=-\frac{1}{2} g_{A B} R_{G B}^{2}+2 R R_{A B}-4 R_{A C} R_{B}^{C}-4 R^{C D} R_{A C B D}+2 R_{A}{ }^{C D E} R_{B C D E} .
$$

While the EGB equation (9) is rather complicated, it can be shown that in factorizable spacetime, the EGB equation can be simplified into a rather illuminating form: after inserting the ansatz (11) into the EGB equations (91), the transverse and longitudinal EGB equations can be simplified to give

$$
\begin{gathered}
\alpha R_{G B}^{2}[g]+R[g]=-\frac{1}{M_{6}^{4}} T_{\gamma}^{B} \\
(\alpha R[g]+1) R[\gamma]+\frac{1}{2} R[g]=-\frac{1}{2 M_{6}^{4}}\left[T_{\gamma}^{B}+T^{b}\right] .
\end{gathered}
$$


From those two equations we can see that the main feature of codimension 2 branes in Einstein gravity is retained in EGB gravity: the scalar curvature of the 4-dimensional spacetime is still determined only by the transverse component of the bulk energy-momentum tensor from the transverse EGB equation (11); the bulk geometry is then determined by the longitudinal equation (12). So the EGB equations are still "factorizable" in factorizable spacetime. Thus in the EGB gravity, we still can move the cosmological constant problem completely into the bulk.

Now we have good motivation to proceed to see how the spacetime solutions will be modified in EGB gravity. As a first remark, it is interesting to see from Eqs.(11) and (12) that the Gauss-Bonnet term couples only with the 4-dimensional scalar curvature $R[g]$. Thus, when the 4-dimensional geometry is flat, EGB equations will always reduce to Einstein equations (5) and (66). So in this case, the bulk solution is the same as the one given in Ref.[6]. Thus what is really interesting is the case when the brane geometry is not flat. In the next section, we will consider de Sitter geometry on the brane.

\section{DE SITTER BRANES IN EINSTEIN-GAUSS-BONNET GRAVITY}

From the recent cosmological observation that our universe is currently accelerating [21], we are interested in solutions with de Sitter geometry on the brane. So we will consider in this section the case that the geometry on the brane is de Sitter, i.e. $R[g]_{\mu \nu}=\Lambda_{4} g_{\mu \nu}$ and $R[g]=4 \Lambda_{4}$, where $\Lambda_{4} \geq 0$ is the 4-dimensional cosmological constant. Under those assumptions of spacetime geometry, it can be seen from Eqs.(11) and (12) that the bulk energy-momentum tensor $T_{A B}^{B}$ must be constant along the bulk.

Let's first discuss the 4-dimensional geometry by Eq.(11). Under the assumption of maximal symmetric, it can be rewritten as

$$
\frac{8}{3} \alpha \Lambda_{4}^{2}+4 \Lambda_{4}=-\frac{1}{M_{6}^{4}} T_{\gamma}^{B}
$$

From Eq.(13), we can find that the 4-dimensional cosmological constant is given in terms of the bulk energy-momentum tensor by

$$
\Lambda_{4}=\frac{3}{4 \alpha}\left[-1 \pm \sqrt{1-\frac{2 \alpha}{3 M_{6}^{4}} T_{\gamma}^{B}}\right] .
$$

Thus, the first different feature we encounter in the EGB gravity is that, for any given $T_{A B}^{B}$, unless it satisfies $T_{\gamma}^{B}=\frac{3 M_{6}^{4}}{2 \alpha}$, we will have two solutions of the brane geometry. After 
the brane geometry is determined, the bulk geometry is uniquely determined by the brane geometry from Eq.(12). Thus, generally, for any given bulk matter content, there will be two different solutions of the EGB equation. This is obviously not a pleasant feature. However, we will argue that the "-" branch of the solution is unphysical and should be discarded. It can be seen from Eq.(14) that for the "-" branch, the coefficient of $R[\gamma]$ in Eq.(12), i.e. $4 \alpha \Lambda_{4}+1$, is always negative; while in Einstein gravity, i.e. $\alpha=0$, it is always positive. This means that for the "-" branch, the gravity in the transverse dimension is repulsive: positive bulk energy density will give rise to negative curvature and only negative tension branes can give rise to a positive deficit angle. We think those properties are too exotic so should be regarded as unphysical. Thus in the following discussions, we will discard the "-" branch.

Then let's discuss the bulk geometry from Eq.(12), which now can be written as

$$
\left(4 \alpha \Lambda_{4}+1\right) M_{6}^{4} R[\gamma]=-\frac{1}{2} T_{g}^{B}-2 M_{6}^{4} \Lambda_{4}+\frac{2 \sigma}{\sqrt{|\gamma|}} \delta^{(2)}(y) .
$$

First, in the case of a vacuum bulk, i.e. $T_{A B}^{B}=0$. From the "+" branch of Eq.(14), we have $\Lambda_{4}=0$, and Eq.(15) will just reduce to Einstein gravity. Thus the bulk geometry will be same as the case discussed in Ref. [19].

Next, let's consider the presence of bulk fields. Following Refs. [6, 7, 20], we expect the extra dimensions to have the topology of a sphere $\mathcal{S}^{2}$. Thus the 2-dimensional metric $\gamma_{a b}$ will be of the form,

$$
\gamma_{a b} d y^{a} d y^{b}=a_{0}^{2}\left(d \theta^{2}+\beta^{2} \sin ^{2} \theta d \varphi^{2}\right)
$$

where $a_{0}$ is the size of the extra dimensions and $\beta$ is related to the deficit angle $\delta$ by $\delta=2 \pi(1-\beta)$.

Transforming the metric (16) into the conformal form,

$$
\gamma_{a b} d y^{a} d y^{b}=\psi(r)\left(d r^{2}+r^{2} d \varphi^{2}\right)
$$

where $\psi$ is given by $[6]$

$$
\psi(r)=\frac{4 \beta^{2} a_{0}^{2}}{r^{2}\left[\left(r / r_{0}\right)^{\beta}+\left(r / r_{0}\right)^{-\beta}\right]^{2}},
$$

and substituting this into Eq.(15), it can be found that $a_{0}$ and $\beta$ are given by

$$
\begin{gathered}
a_{0}^{2}=\frac{M_{6}^{4}\left(1+4 \alpha \Lambda_{4}\right)}{-\frac{1}{4} T_{g}^{B}-M_{6}^{4} \Lambda_{4}}, \\
\beta=1-\frac{\sigma}{2 \pi M_{6}^{4}\left(1+4 \alpha \Lambda_{4}\right)} .
\end{gathered}
$$


Equations (19), (20) and (14) determine the brane and bulk geometry completely. They are the main result of this paper. Below we will discuss mainly its application to the scenario of Ref.[6]. Before that, two remarks are in order about those solutions.

First, a whole new feature of the solution (20) compared to the Einstein case is that the deficit angle in the extra dimensions will now depend on the geometry of the branes. From this, we can find an interesting geometric argument in favor of a positive Gauss-Bonnet coupling constant. In the case of a negative Gauss-Bonnet coupling constant, the geometry will become singular when $\Lambda_{4}>-1 /(4 \alpha)$. Since we expect $\Lambda_{4}$ to be very large during the inflation era, the requirement of a nonsingular geometry forces us to rule out a negative Gauss-Bonnet coupling constant.

Second, the brane geometry in 6-dimensional Einstein-Gauss-Bonnet gravity is also discussed in Ref.[11]. The authors actually considered only the longitudinal component of the EGB equation and concluded that Einstein gravity will restore on the brane. Due to our analysis, the brane geometry is determined by the transverse component of the EGB equation and while the longitudinal equation looks like Einstein equation, it actually determines the bulk geometry after the brane geometry is found by the transverse equation. This can be seen more clearly by the expression for the 4-dimensional cosmological constant in Ref. [11] (Eq.(21) in that reference). Actually, Eq.(21) in Ref.[11] is exactly Eq.(20), from which we can see that it actually determines the deficit angle after the 4-dimensional cosmological constant is found from Eq.(14).

Now, let's discuss a specific example of the solutions (19), (20) and (14). A lot of the recent works on codimension 2 branes are motivated by the exact solution presented by Carroll and Guica [6] which shows explicitly the independence of the 4-dimensional geometry on the brane tension. Thus we think it is most important to discuss the corresponding solutions in EGB gravity and compare it with that of Ref. [6]. The solution presented by Carroll and Guica assumes a bulk cosmological constant and a magnetic flux, which is described by the bulk action [6],

$$
S_{6}=\int d^{6} X \sqrt{|G|}\left(\frac{1}{2} M_{6}^{4} R-\lambda-\frac{1}{4} F_{A B} F^{A B}\right),
$$

where $M_{6}$ is the 6-dimensional reduced Planck mass and $\lambda$ is the 6-dimensional vacuum energy density. The 2-form field strength takes the form $F_{a b}=\sqrt{|\gamma|} B_{0} \epsilon_{a b}$, where $B_{0}$ is a constant and $\epsilon_{a b}$ is the standard antisymmetric tensor. Other components of $F_{A B}$ vanish identically. This model is originally suggested to stabilize the extra dimensions [19, 20]. 
The bulk energy-momentum tensor contains contributions from both the bulk cosmological constant and the gauge field,

$$
T_{A B}^{B}=T_{A B}^{\lambda}+T_{A B}^{F},
$$

for which the explicit forms are

$$
\begin{aligned}
& T_{A B}^{\lambda}=-\lambda\left(\begin{array}{cc}
g_{\mu \nu} & 0 \\
0 & \gamma_{a b}
\end{array}\right) \\
& T_{A B}^{F}=-\frac{1}{2} B_{0}^{2}\left(\begin{array}{cc}
g_{\mu \nu} & 0 \\
0 & -\gamma_{a b}
\end{array}\right) .
\end{aligned}
$$

So we have $T_{1}^{B}=-4 \lambda-2 B_{0}^{2}$ and $T_{2}^{B}=-2 \lambda+B_{0}^{2}$.

At first, we generalize the flat brane solution of Ref.[6] to de Sitter brane, which is given by

$$
\begin{gathered}
a_{0}^{2}=\frac{M_{6}^{4}}{2 \lambda-3 M_{6}^{4} \Lambda_{4}}, \\
\beta=1-\frac{\sigma}{2 \pi M_{6}^{4}}, \\
M_{6}^{4} \Lambda_{4}=\frac{1}{2} \lambda-\frac{1}{4} B_{0}^{2} .
\end{gathered}
$$

It is interesting to note that for the geometry to be nonsingular, from Eq.(24), we must have $\Lambda_{4}<2 \lambda /\left(3 M_{6}^{4}\right)$. However, from Eq.(26) , this is always satisfied. Thus the de Sitter geometry of the brane will never make the bulk geometry singular.

From Eq.(26), we can see that the puzzle of a small 4-dimensional cosmological constant is now transformed to the question of explaining a fine-tuning between the 6-dimensional vacuum energy and the magnetic flux, which is a purely bulk problem. Thus in this scenario the cosmological constant problem is moved completely into the bulk. Of course, this does not solve the cosmological constant problem, but it transforms the nature of the problem in an interesting way. At a first glance, it is tempting to appeal to the usual supersymmetry argument [2] to set both $\lambda$ and $B_{0}^{2}$ very small, thus avoiding fine-tuning between them. However, this cannot work. From Eq.(24), we can see that we must require either $\lambda$ or $B_{0}^{2}$ to be of the order $M_{6}^{6}$ so that the size of the extra dimensions can be phenomenologically viable. Thus, there is a real fine-tuning problem in the bulk. Currently, we still do not know 
whether this fine-tuning can be technically natural. Thus, it would be very interesting that if in the EGB gravity, we can have a way to release this fine-tuning. We will see below that when the Gauss-Bonnet coupling constant is large, this is possible.

Then, we turn to the discussion of solutions in EGB gravity. From Eqs.(19), (201) and (14), the corresponding solution in EGB gravity is given by

$$
\begin{gathered}
a_{0}^{2}=\frac{M_{6}^{4}\left(1+4 \alpha \Lambda_{4}\right)}{2 \lambda-3 M_{6}^{4} \Lambda_{4}-\frac{4}{3} \alpha M_{6}^{4} \Lambda_{4}^{2}}, \\
\beta=1-\frac{\sigma}{2 \pi M_{6}^{4}\left(1+4 \alpha \Lambda_{4}\right)}, \\
\Lambda_{4}=\frac{3}{4 \alpha}\left(\sqrt{1+\frac{2 \alpha}{3} \frac{2 \lambda-B_{0}^{2}}{M_{6}^{4}}}-1\right) .
\end{gathered}
$$

As a first remark, while we have discussed above, a negative $\alpha$ may result in a singular spacetime; for a positive $\alpha$, while it is not very obvious, it still can be shown that the geometry is always nonsingular by an argument that is similar to the Einstein case.

Then let's discuss the cosmological constant problem in EGB gravity as expressed by Eq. (29). Since we generally have $\lambda<M_{6}^{6}$ and $B_{0}^{2}<M_{6}^{6}$, so when $\alpha M_{6}^{2}<1$, we have $\alpha\left(2 \lambda-B_{0}^{2}\right) / M_{6}^{4} \ll 1$. Expanding the RHS of Eq.(29) to first order, we can find that $\Lambda_{4} \sim\left(2 \lambda-B_{0}^{2}\right) / M_{6}^{4}$. Thus, in this case we are actually facing the same fine-tuning as in Einstein gravity in order to get a small cosmological constant. This is not a surprise, since it is natural for the solution to reduce to the Einstein case when $\alpha$ is small. So what is interesting is the case where the Gauss-Bonnet coupling constant is large. Let's assume $2 \lambda-B_{0}^{2} \sim M_{6}^{6}$, i.e. we do not have a fine-tuning in the bulk, and when $\alpha M_{6}^{2} \gg 1$, i.e. considering the case of a large Gauss-Bonnet coupling constant, from Eq.(29), we can obtain

$$
\Lambda_{4} \sim \frac{M_{6}}{\sqrt{\alpha}} .
$$

Thus even if we do not have a fine-tuning in the bulk, for a sufficiently large $\alpha$, we still can get a small 4-dimensional cosmological constant. In this case, the current cosmological expansion acceleration is actually driven by the 6-dimensional Gauss-Bonnet term, which is in some sense similar to the recent model of $1 / R$ gravity proposed by Carroll et al. [22]: the current cosmological expansion acceleration is driven by a $1 / R$ term in the 4 -dimensional gravitational lagrangian. Of course, in order for the $\Lambda_{4}$ to be the order of the observational 
value, $\alpha^{-1}$ also needs to be fine-tuned to an extremely small value. Thus in the current case, we have actually traded the fine-tuning in the bulk to a fine-tuning in the Gauss-Bonnet coupling constant. Although this new fine-tuning also seems unnatural now, the cosmological constant problem is so hard to solve that it is worth transforming it to a new problem for further investigations. Furthermore, this shows the qualitative feature of what will happen if we consider higher-derivative gravity in the bulk. Maybe considering more complicated higher-derivative gravity theories, such as forth order combinations of the curvature tensor can further release the fine-tuning in a more natural way. This deserves further investigating. It is worth mentioning that similar fine-tuning problem also happens in the $1 / R$ gravity: the coefficient of the $1 / R$ term should also be extremely small to account for the current cosmic accelerating expansion 22]. In the $1 / R$ gravity, this is unnatural from an effective field point of view and can lead to some inconsistencies when the theory is treated quantum

mechanically [23]. Now we still do not know whether similar problem will be presenting here.

As a final remark, in Ref.[24], Navarro considered using a 4-form field in place of the 2-form field in the action (21). By using Eqs.(19), (20) and (14), it is trivial to generalize Navarro's solution to the EGB gravity.

\section{CONCLUSIONS AND DISCUSSIONS}

In this paper, we have discussed the gravitational properties of codimension 2 branes in Einstein-Gauss-Bonnet gravity and their implications in addressing the cosmological constant problems.

Although the current scenario is originally introduced to discuss the cosmological constant problem, it is also mandatory that cosmological models from String Theory should be reconciled with inflation, now a quite well-established ingredient of modern cosmology 27] (see, e.g., Ref. 25] for a recent review of braneworld inflation; inflation in 5-dimensional EGB gravity is recently discussed in Ref.[26]). When considering inflation model building in the present scenario, an observation is that the inflaton must be a bulk field. This is in sharp contrast to the discussions of the codimension 1 case, where most of the inflation model assumes the inflaton to be confined on the brane [25]. The reason for this is simple. Current observation of the CMB power spectrum tells us that during inflation, the energy density 
of inflaton should be almost constant [27]. Thus, if the inflaton is a field confined on the brane, then during inflation it will behave just like the brane tension. So the above analysis tells us that it cannot affect the 4-dimensional geometry. On the other hand, if the inflaton is a bulk field, then its effects during inflation are just equivalent to a renormalization of the 6-dimensional cosmological constant $\lambda$.

So in the Einstein gravity case, the Hubble parameter during inflation $H^{2} \equiv \Lambda_{4} / 3$ will be given from Eq.(26) by

$$
H^{2} \sim V / M_{6}^{4}
$$

where $V$ is the potential of the bulk inflaton field. Thus the energy scale of the potential would be of order $\left(H / M_{6}\right)^{1 / 3} M_{6}$ during inflation. In the original model of large extra dimensions [1], in order to address the gauge hierarchy problem, the 6-dimensional reduced Planck mass is assumed at most a few orders higher than the supersymmetry breaking scale which is of order $1 \mathrm{TeV}$. On the other hand, current $\mathrm{CMB}$ data prefers a high inflation scale which is at most several orders of magnitude smaller than the GUT scale $\sim 10^{16} \mathrm{GeV}$ [27]. Thus, the potential $V$ during inflation is necessarily larger than $M_{6}$. If the inflaton is a brane field, there is nothing unnatural here. But as we have commented above, inflaton must be a bulk field now. So it is very unnatural for a bulk field to have an energy scale larger than the bulk Planck mass. Therefore, implementing successful inflation scenario encounters fundamental difficulties in codimension 2 brane scenarios [31].

The situation is worse in EGB gravity. From Eq.(29), the Hubble parameter during inflation will be given by Eq.(31) when $M_{6}^{2} \alpha \ll 1$ and it reduces to the Einstein case. When $M_{6}^{2} \alpha \gg 1$, from Eq.(30), the Hubble parameter will be given by

$$
H^{2} \sim \frac{M_{6}}{\sqrt{\alpha}} \sqrt{V}
$$

Thus the energy scale of the potential would be of order $\left(H / M_{6}\right)^{2 / 3}\left(\alpha M_{6}^{2}\right)^{1 / 6} M_{6}$ during inflation. Then a higher potential energy is needed compared with the Einstein case (31) to implement the inflation. This makes the problem we discussed above more severe.

Faced with the above problem, it is worth considering other mechanism of driving an inflation on the brane rather than a bulk scalar field. A seemingly promising candidate is the $R^{2}$ inflationary model of Starobinsky [28]. However, in order to avoid the above problem, we assume that the $R^{2}$ term is only induced on the brane, like the case of induced gravity model given by Dvali et al. [29]. More concretely, we may consider adding to the bulk 
lagrangian (21) an induced $R^{2}$ term,

$$
S_{\text {induced }}=\int d^{4} x \sqrt{|g|} \tilde{\alpha} R[g]^{2},
$$

where $\tilde{\alpha}$ will be of order $M_{4}^{-2}$ [28]. It is worth commenting that such a term may be induced by quantum effects of conformal fields on the brane, and $R^{2}$ inflation on codimension 1 braneworld has been discussed in Ref. [30]. This and other possibilities to handle the inflation model building problems deserve further investigation.

\section{Acknowledgments}

PW would like to thank Sergei D. Odintsov, Jeremie Vinet and Liu Zhao for helpful comment on the manuscript. XHM would also like to express his thanks to the Physics Department of UoA for its hospitality extended to him. This work is supported partly by an ICSC-World Laboratory Scholarship, a China NSF and Doctoral Foundation of National Education Ministry.

[1] N. Arkani-Hamed, S. Dimopoulos and G. Dvali, Phys. Lett. B 429 (1998) 263 hep-ph/9803315; I. Antoniadis, N. Arkani-Hamed, S. Dimopoulos and G. Dvali, Phys. Lett. B 436 (1998) 257 hep-ph/9804398; N. Arkani-Hamed, S. Dimopoulos and J. March-Russell, Phys. Rev. D 63 (2001) 064020 hep-th/9809124; J. Vinet and J. M. Cline, hep-th/0406141.

[2] S. M. Carroll, Living Rev. Rel. 4 (2001) 1 astro-ph/0004075.

[3] S. Weinberg, Rev. Mod. Phys. 61 (1989) 1.

[4] G. Dvali, G. Gabadadze, M. Shifman, Phys. Rev. D 67 (2003) 044020 hep-th/0202174.

[5] N. Arkani-Hamed, S. Dimopoulos, N. Kaloper and R. Sundrum, Phys. Lett. B 480 (2000) 193 hep-th/0001197; S. Kachru, M. B. Schulz and E. Silverstein, Phys. Rev. D 62 (2000) 045021 hep-th/0001206; S. Kachru, M. B. Schulz and E. Silverstein, Phys. Rev. D 62 (2000) 085003 hep-th/0002121; S. M. Carroll and L. Mersini, Phys. Rev. D 64 (2001) 124008 hep-th/0105007.

[6] S. M. Carroll and M. M. Guica, hep-th/0302067.

[7] I. Navarro, JCAP 0309 (2003) 004 hep-th/0302129; J.-W. Chen, M. A. Luty and E. Ponton, JHEP 0009 (2000) 012 hep-th/0003067; A. Kehagias, hep-th/0406025 Z. C. Wu, 
hep-th/0405249 R. Gregory, JHEP 0306 (2003) 041 hep-th/0304262; R. Gregory and C. Santos, Class. Quant. Grav. 20 (2003) 21 hep-th/0208037; M.L. Graesser, J. E. Kile, P. Wang, hep-th/0403074 I. Navarro and J. Santiago, JHEP 0404 (2004) 062 hep-th/0402204; R. A. Battye, B. Carter, A. Mennim, Phys. Rev. Lett. 92 (2004) 201305 hep-th/0312198; J. Louko and D. L. Wiltshire, JHEP 0202, 007 (2002) hep-th/0109099; J. Garriga and M. Porrati, hep-th/0406158.

[8] G.W. Gibbons and D. L. Wiltshire, Nucl. Phys. B 287 (1987) 717 hep-th/0109093.

[9] B. Carter, Int. J. Theor. Phys. 40 (2001) 2099 gr-qc/0012036.

[10] J. M. Cline, J. Descheneau, M. Giovannini and J. Vinet, JHEP 0306 (2003) 048 hep-th/0304147.

[11] P. Bostock, R. Gregory, I. Navarro and J. Santiago, Phys. Rev. Lett. 92 (2004) 221601 hep-th/0311074.

[12] J. Vinet and J. M. Cline, hep-th/0406141

[13] O. Corradini, A. Iglesias, Z. Kakushadze and P. Langfelder, Phys. Lett. B 521 (2001) 96 hep-th/0108055.

[14] O. Corradini, A. Iglesias, Z. Kakushadze and P. Langfelder, Mod. Phys. Lett. A 17 (2002) 795 hep-th/0201201; O. Corradini, A. Iglesias and Z. Kakushadze, Int. J. Mod. Phys. A 18 (2003) 3221 hep-th/0212101; O. Corradini, A. Iglesias and Z. Kakushadze, Mod. Phys. Lett. A 18 (2003) 1343 hep-th/0305164; A. Iglesias and Z. Kakushadze, hep-th/0306297.

[15] Y. Duan and X. H. Meng, J. Math. Phys. 34 (1993) 1149

[16] S. Nojiri and S. D. Odintsov, JHEP 0007 (2000) 049; S. Nojiri, S. D. Odintsov and S. Ogushi, Int. J. Mod. Phys. A16 (2001) 5085; C. Charmousis and J.Dufaux, Class. Quant. Grav. 19 (2002) 4671 hep-th/0202107; S.C.Davis, Phys. Rev. D 67 (2003) 024030 hep-th/0208205; S. Nojiri, S. D. Odintsov and S. Ogushi, Phys. Rev. D 65 (2002) 023521; J. E. Lidsey, S. Nojiri and S. D. Odintsov, JHEP 06 (2002) 026; J. E. Kim, B. Kyae and H. M. Lee, Phys. Rev. D 62 (2000) 045013; Y. M. Cho, I. P. Neupane and P. S. Wesson, Nucl. Phys. B 621 (2002) 388 hep-th/0104227; I. P. Neupane, Phys. Lett. B 512 (2001) 137 hep-th/0104226; I. P. Neupane, Class. Quant. Grav. 19 (2002) 5507 hep-th/0106100; Y. M. Cho and I. P. Neupane, Int. J. Mod. Phys. A 18 (2003) 2703 hep-th/0112227.

[17] B.Zweibach, Phys. Lett. B 156 (1985) 315.

[18] S. Kanno and J. Soda, hep-th/0404207, H. M. Lee and G. Tasinato, JCAP 0404 (2004) 009 
hep-th/0401221; M. Giovannini and H. Meyer, Phys. Rev. D64 (2001) 124025.

[19] R. Sundrum, Phys. Rev. D 59, 085010 (1999) hep-ph/9807348.

[20] P. G. Freund and M. A. Rubin, Phys. Lett. B 97 (1980) 233; S. M. Carroll, J. Geddes, M. B. Hoffman and R. M. Wald, Phys. Rev. D 66, 024036 (2002) hep-th/0110149; U. Guenther and A. Zhuk, Phys. Rev. D 56 (1997) 6391 gr-qc/9706050; ibid, Class. Quant. Grav. 15 (1998) 2025 gr-qc/9804018; ibid, Phys. Rev. D 61 (2000) 124001 hep-ph/0002009; ibid, Class. Quant. Grav. 181441 hep-ph/0006283; U. Guenther, A. Starobinsky and A. Zhuk, Phys. Rev. D 69 (2004) 044003 hep-ph/0306191; U. Guenther, P. Moniz and A. Zhuk, Phys. Rev. D 68 (2003) 044010 hep-th/0303023.

[21] A. Riess et al. Astron. J. 116 (1998) 1009 astro-ph/9805201; S. Perlmutter el al. Astroph. J. 517 (1999) 565 astro-ph/9812133.

[22] S. M. Carroll, V. Duvvuri, M. Trodden and M. S. Turner, astro-ph/0306438, S. Capozziello, S. Carloni and A. Troisi, astro-ph/0303041; S. Nojiri and S. D. Odintsov,Phys. Lett. B 576 (2003) 5 hep-th/0307071; D. N. Vollick, Phys. Rev. D 68 (2003) 063510; X.H.Meng and P.Wang, Class. Quant. Grav. 21 (2004) 951 astro-ph/0308031; E. E. Flanagan, Phys. Rev. Lett. 92 (2004) 071101 astro-ph/0308111.

[23] E. E. Flanagan, gr-qc/0403063.

[24] I. Navarro, Class. Quant. Grav. 20 (2003) 3603 hep-th/0305014.

[25] J. E. Lidsey, astro-ph/0305528

[26] J. E. Lidsey and N. J. Nunes, Phys. Rev. D67 (2003) 103510 astro-ph/0303168; X. Meng and P. Wang, Class. Quant. Grav. 21 (2004) 2527 hep-ph/0312113; B. C. Paul, M. Sami, Phys. Rev. D 70 (2004) 027301 hep-th/0312081; J.-F. Dufaux, J. Lidsey, R. Maartens and M. Sami, hep-th/0404161, S. Tsujikawa, M. Sami and R. Maartens, astro-ph/0406078.

[27] M. Trodden and S. M. Carroll, astro-ph/0401547.

[28] A. A. Starobinsky, Phys. Lett. B 91 (1980) 99.

[29] G. Dvali, G. Gabadadze and M. Porrati, Phys. Lett. B 485 (2000) 208 hep-th/0005016.

[30] S. Nojiri, S. D. Odintsov and S. Zerbini, Phys. Rev. D 62 (2000) 064006 hep-th/0001192; S. Nojiri and S. D. Odintsov, Phys. Lett. B 484 (2000) 119 hep-th/0004097.

[31] If considering warped extra dimensions, it is possible to get any value of 4-dimensional cosmological constant by tuning the warp factor without a large potential in the bulk [10], thus avoiding the problem discussed above. We thank J. Vinet for pointing this possibility to us. 
However, in this case, 4-dimensional cosmological constant is actually only an integration constant and cannot be determined from the model parameter. We think this seems not a very good feature so we do not consider this possibility further in this work while this possibility of course deserves further detailed considerations. 